\title{
Transformer-ratio optimization in nonlinearly driven hollow plasma channels
}

\author{
J. P. Farmer and A. Pukhov \\ Institut für Theoretische Physik I, Universität Düssseldorf, Düsseldorf 40225 Germany
}

(Received 9 November 2018; published 11 February 2019)

\begin{abstract}
Acceleration to high energies in electron-driven wakefield accelerators requires a carefully tailored drive beam. High driver currents can, however, modify the accelerating structure response, shifting the optimal driver profile. In this work we present a simple model to describe this nonlinear beamloading in hollow plasma channels. The increase in channel radius and the generation of a return current in the bulk plasma act to modify the resonant frequency and accelerator loss factor. Modeling these effects allows the optimal driver profile to be calculated.
\end{abstract}

DOI: 10.1103/PhysRevAccelBeams.22.021003

Plasma is an attractive accelerating medium due to the high field gradients it can support. A driver may be used to excite a wakefield, which in turn accelerates a trailing witness bunch [1]. The driver may be a laser [2] or a charged particle beam [3].

For a laser driver, acceleration is limited by dephasing between the driver and witness, although this can be overcome by staging [4]. For a charged beam, the energy gain is limited by driver energy and the transformer ratiothe ratio of the accelerating field acting on the witness to the decelerating field acting on the driver. A large transformer ratio is not a concern for proton-driven acceleration, where the driver energy is high [5,6]. For an electron driver, however, a high transformer ratio is necessary to achieve high energies in a single stage.

A high transformer ratio requires a long driver with a carefully chosen current profile [7]. One may use, for example a ramped density bunch $[8,9]$ or a ramped bunch train [10] to control the excited wakefield. This wakefield must be controlled over the entire acceleration length to accelerate the witness to high energies.

In homogeneous plasma, acceleration with a long drive beam is limited by the transverse two-stream instability [11]. Different modes of the instability dominate for different beam parameters [12] and manifest as different effects, including self modulation [13,14] and hosing [15].

Self modulation may be avoided by instead using a a hollow plasma channel. The configuration in the co-moving frame $\tau=t-z / c$ is shown in Fig. 1. A driver propagating through the channel excites a wakefield. There are no

Published by the American Physical Society under the terms of the Creative Commons Attribution 4.0 International license. Further distribution of this work must maintain attribution to the author(s) and the published article's title, journal citation, and DOI. focusing forces inside the channel, preventing modulation of the beam. Hosing, unfortunately, persists, as the drive beam is attracted to the channel walls [16,17], leading to beam breakup (BBU). Recent work has, however, shown that stable acceleration can be achieved in a hollow-channel geometry by making use of a coaxial plasma filament [18], opening the possibility for high-energy acceleration.

To fully exploit plasma as an accelerating medium, high drive currents are required to generate large wakefields. However, a high-current driver will modify the channel response, changing the wakefield. In this work, we develop a simple analytic model to describe this nonlinear beamloading. This allows the optimized beam profile for a highcurrent driver to be calculated, allowing acceleration to high energies over short acceleration distances.

To illustrate the effect of nonlinear beamloading, simulations were carried out with the fully three-dimensional quasi-static particle-in-cell code qv3d [19], developed on the VLPL platform [20]. Figure 2(a) shows the normalized wakefield excited by a flat-top drive beam propagating along the axis of a hollow channel of radius $R=k_{p} r=1$, for a drive current of $0.1,1$ and $10 \mathrm{kA}$. Here, $k_{p}=\omega_{p} / c$, where $\omega_{p}=\left(n_{e} e^{2} / \varepsilon_{0} m\right)^{1 / 2}$ is the bulk plasma frequency, with $n_{e}$ the unperturbed plasma-electron number density. A cell size of $0.02 \times 0.02 \times 0.1 k_{p}^{-1}$ is used, with 64 and 4 particles per cell in the drive beam and bulk plasma, respectively. The transverse size of the simulation box is $12 k_{p}^{-1}$ to correctly model the resonant channel frequency [21]. As can be seen, an increase in the driver current is accompanied by marked shift in both the frequency and relative amplitude of the excited wakefield.

For a tailored drive beam, this will result in a shift in the channel response along the driver length, impacting on the transformer ratio. This is illustrated in Fig. 2(b), which shows the excited wakefield for a double-triangular (DT) drive beam [9]. For low current, this profile gives a constant 


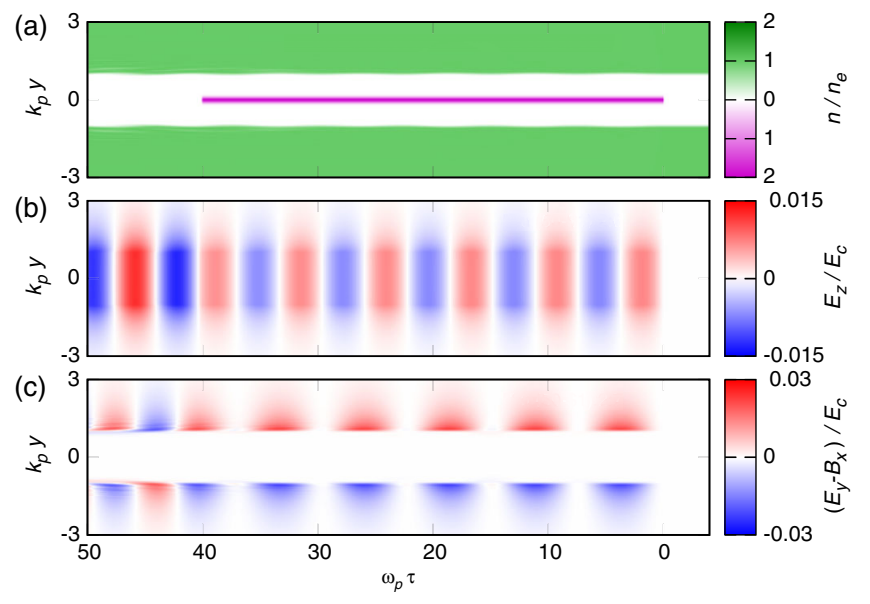

FIG. 1. Geometry of the hollow plasma channel. (a) A drive beam (purple) propagates left-to-right through a hollow channel. The response of the bulk plasma (green) generates (b) longitudinal and (c) transverse fields. $E_{c}=m c \omega_{p} / e$ is the critical field.

decelerating field along most of the driver, as expected from linear theory. However, for higher current, the decelerating field increases along the driver length. For the $10 \mathrm{kA}$ driver, the decelerating field increases by $28 \%$, corresponding to a $22 \%$ decrease in transformer ratio. Understanding nonlinear beamloading is therefore vital to correctly tailor the drive beam to achieve high transformer ratios simultaneously with high accelerating fields.

Schroeder et al. [17] derived the wakefield modes excited by an ultrarelativistic charge passing through a hollow plasma channel. The frequency and amplitude of the $m$ th mode are governed by the corresponding channel
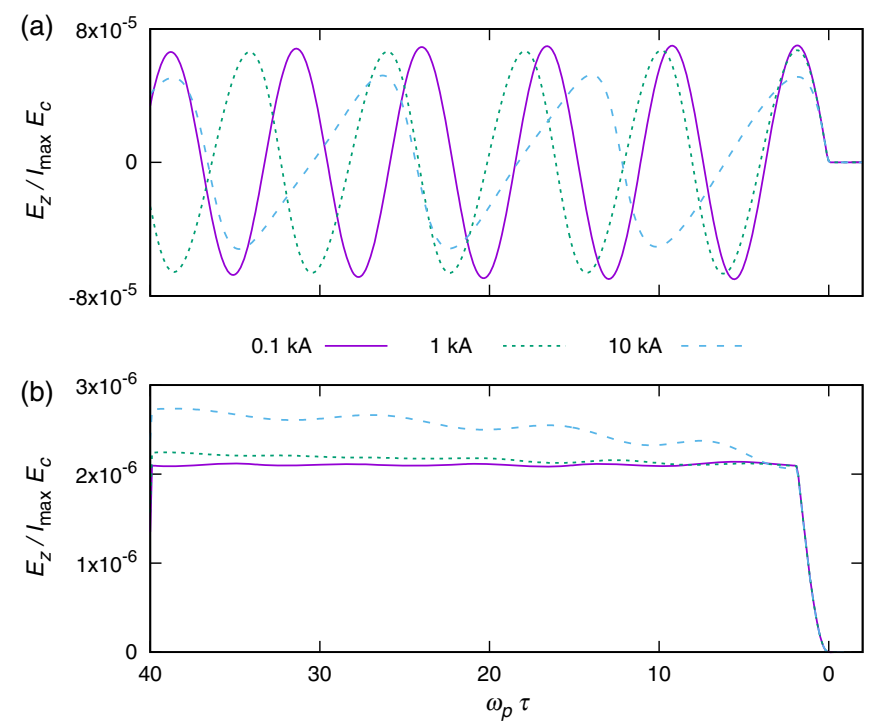

FIG. 2. On-axis longitudinal electric field normalized to the peak driver current in a channel of radius $R=1$ for (a) a flat-top and (b) a double-triangular (DT) driver profile, for different peak currents. As the current increases, there is a decrease in both the resonant channel frequency and the wakefield amplitude. frequency and accelerator loss factor, $\omega_{m}$ and $\kappa_{m}$. The accelerator loss factor relates the energy per unit length stored in the structure to the on-axis accelerating field, and serves as a measure of the coupling between driver and channel. These may be simplified without loss of generality to:

$$
\begin{gathered}
\frac{\omega_{m}^{2}}{\omega_{p}^{2}}=\frac{\left(1+\delta_{m 0}\right)(m+1) K_{m+1}(R)}{R K_{m+2}(R)}, \\
\frac{c^{2} \kappa_{m}}{\omega_{p}^{2}}=\frac{2(m+1) K_{m}(R)}{R^{2} K_{m+2}(R)},
\end{gathered}
$$

where $K_{m}$ is the $m$ th-order modified Bessel function of the second kind, and $\delta_{m 0}=1$ for $m=0$ and zero otherwise. The response to a drive beam can then be obtained by integrating over all charges, accurately reproducing the wakefield in the low-current limit. We here seek a simple extension to these equations to model the shift in frequency and amplitude of the excited wakefield observed for a highcurrent driver. This in turn will provide the optimal driver profile to compensate the effect of nonlinear beamloading, maximizing the attainable transformer ratio.

The most obvious perturbation to the channel due to a high-current driver is the increase in channel radius, as shown in Fig. 3(a) for a $10 \mathrm{kA}$ flat-top beam. The channel radius will oscillate around an equilibrium point, $R_{\mathrm{eq}}$, determined by the driver current. We estimate this equilibrium radius by assuming it depends only on the driver

(a)
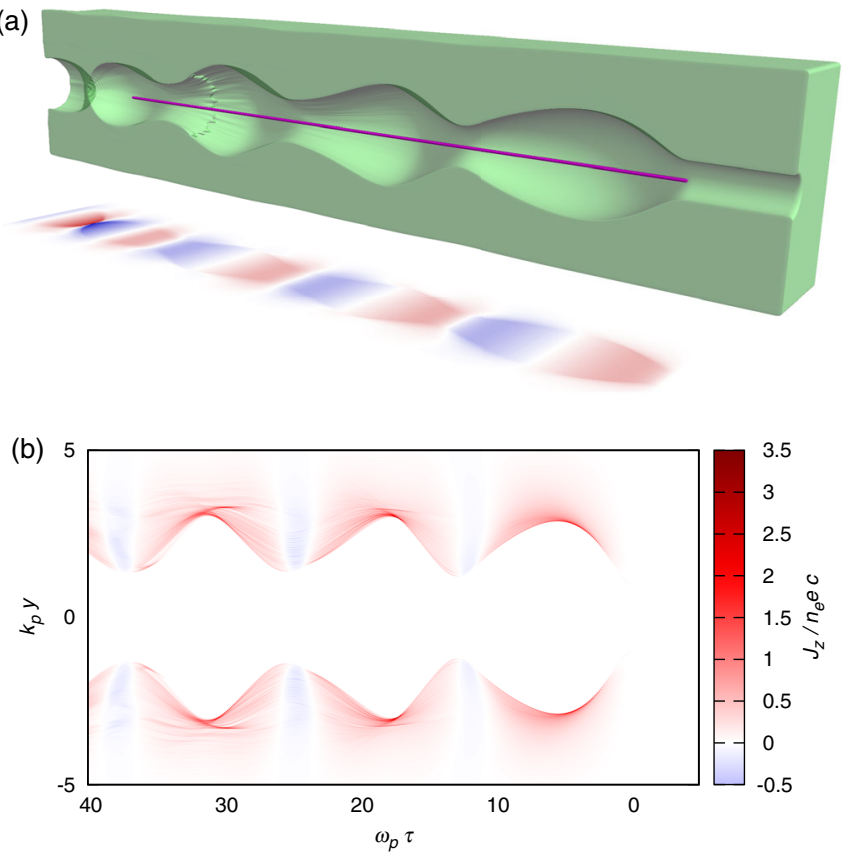

FIG. 3. For high driver current, the channel is perturbed. (a) The channel radius and (b) the bulk plasma return current for a $10 \mathrm{kA}$ flat-top driver. 
current at that point. Equilibrium requires that the enclosed ionic charge cancels the drive-beam charge. For a driver current $I$ in a channel of initial radius $R_{0}$, and assuming stationary ions, this gives:

$$
R_{\mathrm{eq}}=\sqrt{R_{0}^{2}+\frac{4 I}{I_{A}}},
$$

where $I_{A}=4 \pi \varepsilon_{0} m c^{3} / e=17 \mathrm{kA}$ is the natural current unit. Figure 4(a) shows this variation in equilibrium radius as a function of driver current for channels of initial radius $R_{0}=1$ and 3 . However, this increase in channel radius alone is insufficient to explain the change in the excited wakefield.

In addition to the increase in channel radius, a highcurrent beam will also drive a return current in the bulk plasma, as shown in Fig. 3(b). The resulting velocity of the bulk plasma acts to stretch the periodicity of the channel wall oscillations, decreasing the resonant frequency as observed from the beam frame. To calculate the equilibrium value for this bulk plasma velocity, $v_{\text {eq }}$, we assume the return current is equal in magnitude to the beam current at that point, and is uniformly distributed over a skin depth from the perturbed equilibrium radius. This gives:

$$
\frac{v_{\mathrm{eq}}}{c}=-\frac{4 I}{I_{A}} /\left(2 R_{\mathrm{eq}}+1\right) .
$$

The dependence of $v_{\text {eq }}$ on the driver current is shown in Fig. 4(b).

The resonant channel frequency will be modified both by the equilibrium channel radius and the bulk plasma
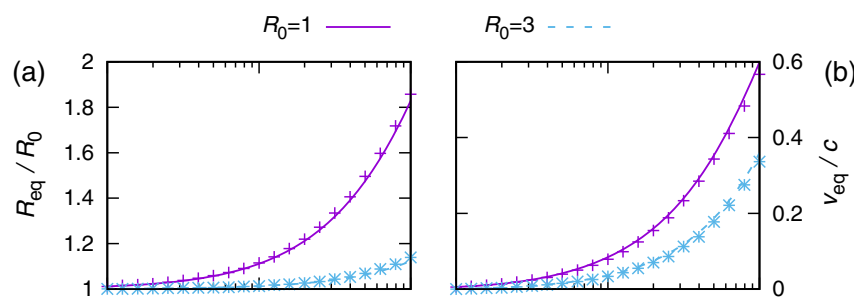

(c)
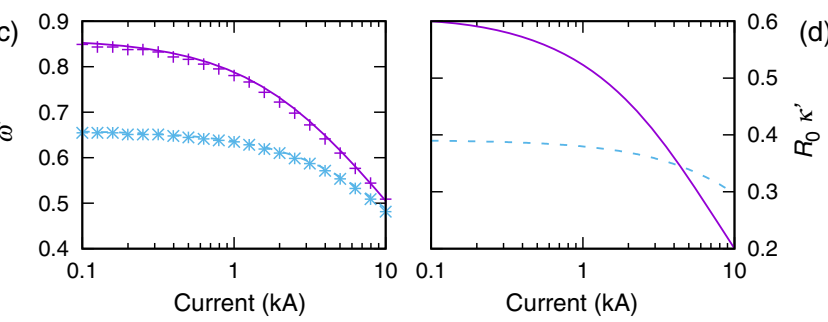

FIG. 4. Plots showing the modified channel response predicted by the simple model presented in Eqs. (3)-(6). (a) Equilibrium channel radius $R_{\text {eq }}$, (b) equilibrium bulk-plasma velocity $v_{\text {eq }}$, (c) modified channel frequency $\omega^{\prime}$, and (d) modified accelerator loss factor $\kappa^{\prime}$. (a-c) additionally show comparison with qv3d simulations (crosses). velocity. The coupling between the driver and the channel will decrease due to the increased channel radius, i.e. a lower loss factor. Since the longitudinal field generated by a drive beam propagating close to the channel axis is dominated by the zeroth-order wakefield mode, we here neglect higher-order modes. The modified frequency and accelerator loss factor are then:

$$
\begin{gathered}
\omega^{\prime}=\omega_{0}\left(R_{\mathrm{eq}}\right) /\left(1-v_{\mathrm{eq}} / c\right), \\
\kappa^{\prime}=\kappa_{0}\left(R_{\mathrm{eq}}\right) .
\end{gathered}
$$

The dependence of the frequency and accelerator loss factor on the driver current are shown in Figs. 4(c) and 4(d), respectively.

To gauge the merit of the model, comparisons are made with qv3d simulations for a flat-top driver for driver currents ranging from 0.1 to $10 \mathrm{kA}$. The equilibrium radius is found by measuring the channel radius where the on-axis field reaches a maximum. The average bulk plasma current over the entire simulation box width is measured over one period of the on-axis electric field, and the corresponding velocity calculated from the measured equilibrium radius. The period of the on-axis field is also used to calculate the channel frequency. Despite the simplicity of the model, it gives excellent agreement with simulations over a wide parameter range, as can be seen in Fig. 4.

This simple model does not reproduce the anharmonic nature of the wakefield for high driver currents seen in Fig. 2(a). This behavior arises due to the oscillation of the channel radius and plasma velocity around their equilibrium values. As a result, no comparison is made for the accelerator loss factor in Fig. 4(d), as the energy stored in the channel depends on both the amplitude and relative phase of the upstream wake excitations. If desired, the model could be further extended to include these oscillations, allowing the wakefield to be accurately modeled for arbitrary driver shape. However, such a model is not necessary to optimize the drive beam.

In the low-current limit, drive beams tailored to deliver high transformer ratio simultaneously act to minimize the transverse channel oscillation along the driver length, providing a constant decelerating field. We therefore assume that drive beams optimized to compensate nonlinear beamloading will similarly minimize the oscillation in both the radius and the bulk plasma current, removing the need for an anharmonic treatment.

As discussed above, nonlinear beamloading leads to an increase in the decelerating field along the length of a DT driver. The density gradient of the optimized driver should therefore decrease along its length. The coupling between the drive beam and the plasma channel is proportional to $\sqrt{\kappa^{\prime}}$. The relation between the DT current profile, $I_{\mathrm{DT}}$, and this scaled-double-triangular (SDT) profile is therefore: 

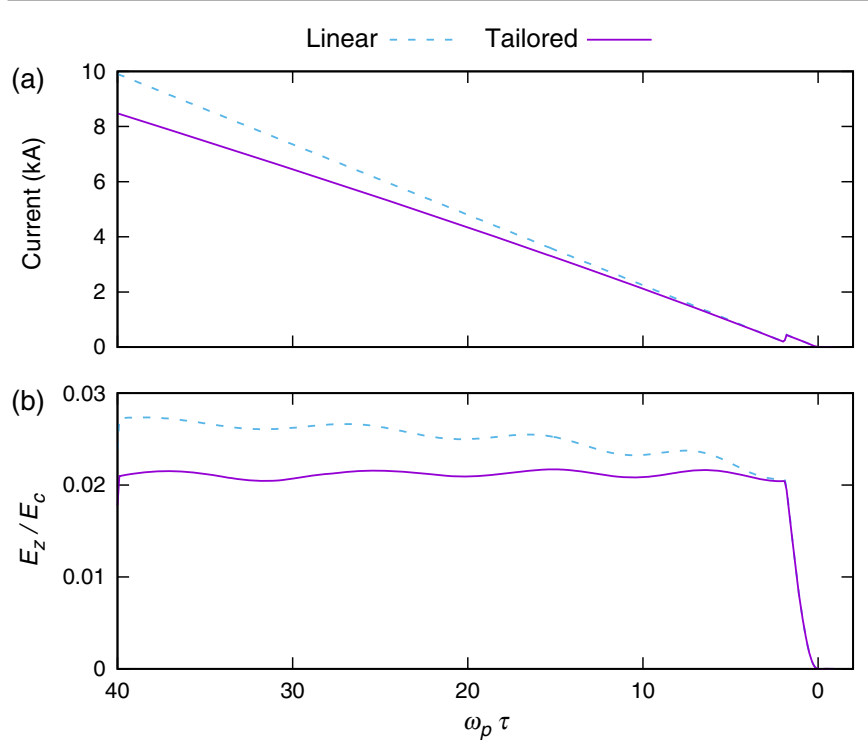

FIG. 5. Taking nonlinear beamloading into account allows the optimal driver current gradient to be calculated. (a) the driver current profile for a DT and scaled-DT (SDT) driver, and (b) the corresponding wakefield. Using the scaled driver allows a nearconstant decelerating field to be obtained, increasing the attainable transformer ratio.

$$
\frac{\partial \sqrt{\kappa^{\prime}} I_{\mathrm{SDT}}}{\partial \omega^{\prime} \tau}=\frac{\partial \sqrt{\kappa_{0}\left(R_{0}\right)} I_{\mathrm{DT}}}{\partial \omega_{0}\left(R_{0}\right) \tau} .
$$

Substitution of Eqs. (1) through (6) into Eq. (7) results in a rather unwieldy expression. Empirical approximations may be possible for specific parameter ranges [18], but not without a loss of generality. The optimal driver profile is, however, readily evaluated numerically using finite difference methods. Figure 5(a) shows the current profile of a DT [as in Fig. 2(b)] and corresponding SDT driver. As can be seen from Fig. 5(b), the use of the scaled driver removes the increase in decelerating field along the driver length, which in turn maximizes the transformer ratio.

The use of the equilibrium values for the radius and return current limit the applicability of this model to long beams. As discussed above, the model could be further developed to calculate the anharmonic plasma wakefield. This would additionally allow the treatment of a short driver, a train or short bunches, or a short witness bunch, for which the use of equilibrium values is not appropriate.

Although not treated here, the shift in the resonant frequency along the driver length will also contribute to the beam stability, as detuning between the driver and channel will lower the BBU growth rate. This is similar to the case of acceleration in the blowout regime, where the shift in resonance along the bubble length leads to a greatly reduced BBU growth rate $[22,23]$. However, the shift in resonance observed in that case is entirely due to the varying bubble radius - here the detuning is dominated by the bulk plasma return current.
In conclusion, we have developed a simple extension to the analytical model for wake excitation in a hollow plasma channel, allowing the treatment of nonlinear beamloading for a long driver. The increase in the channel radius and the return current in the bulk plasma both contribute. Despite its relative simplicity, the model accurately reproduces the shift in resonant frequency over a wide parameter space. This allows the optimal driver gradient to be calculated, allowing high-gradient acceleration to be achieved simultaneously with high-transformer ratios, necessary for high energy gain to be realized.

This work has been supported by the Deutsche Forschungsgemeinschaft and by BMBF.

[1] T. Tajima and J. M. Dawson, Laser Electron Accelerator, Phys. Rev. Lett. 43, 267 (1979).

[2] A. Pukhov and J. Meyer-ter Vehn, Laser wake field acceleration: The highly non-linear broken-wave regime, Appl. Phys. B 74, 355 (2002).

[3] I. Blumenfeld, C. E. Clayton, F.-J. Decker, M. J. Hogan, C. Huang, R. Ischebeck, R. Iverson, C. Joshi, T. Katsouleas, N. Kirby, W. Lu, K. A. Marsh, W. B. Mori, P. Muggli, E. Oz, R. H. Siemann, D. Walz, and M. Zhou, Energy doubling of $42 \mathrm{GeV}$ electrons in a metre-scale plasma wakefield accelerator, Nature (London) 445, 741 (2007).

[4] S. Steinke, J. van Tilborg, C. Benedetti, C. G. R. Geddes, C. B. Schroeder, J. Daniels, K. K. Swanson, A. J. Gonsalves, K. Nakamura, N. H. Matlis, B. H. Shaw, E. Esarey, and W. P. Leemans, Multistage coupling of independent laser-plasma accelerators, Nature (London) 530, 190 (2016).

[5] A. Caldwell, K. Lotov, A. Pukhov, and F. Simon, Protondriven plasma-wakefield acceleration, Nat. Phys. 5, 363 (2009).

[6] E. Adli et al. (AWAKE collaboration), Acceleration of electrons in the plasma wakefield of a proton bunch, Nature (London) 561, 363 (2018).

[7] S. S. Baturin and A. Zholents, Upper limit for the accelerating gradient in the collinear wakefield accelerator as a function of the transformer ratio, Phys. Rev. Accel. Beams 20, 061302 (2017).

[8] K. L. F. Bane, P. Chen, and P. B. Wilson, On collinear wake field acceleration, IEEE Trans. Nucl. Sci. 32, 3524 (1985); also published in Stanford Linear Accelerator Center Report No. SLAC-PUB 3662, 1985.

[9] B. Jiang, C. Jing, P. Schoessow, J. Power, and W. Gai, Formation of a novel shaped bunch to enhance transformer ratio in collinear wakefield accelerators, Phys. Rev. ST Accel. Beams 15, 011301 (2012).

[10] P. Schutt, T. Weiland, and V. M. Tsakanov, in Proceedings of the Second All-Union Conference on New Methods of Charged Particle Acceleration (Springer, New York, 1989), Vol. 7, p. 12.

[11] J. D. Lawson, The Physics of Charged-Particle Beams (Clarendon Press, United Kingdom, 1977). 
[12] J. Krall and G. Joyce, Transverse equilibrium and stability of the primary beam in the plasma wake-field accelerator, Phys. Plasmas 2, 1326 (1995).

[13] K. V. Lotov, in Proceedings of the 6th European Particle Accelerator Conference, Stockholm, 1998 (IOP, London, 1998), pp. 806-808.

[14] N. Kumar, A. Pukhov, and K. Lotov, Self-Modulation Instability of a Long Proton Bunch in Plasmas, Phys. Rev. Lett. 104, 255003 (2010).

[15] D. H. Whittum, Transverse two-stream instability of a beam with a Bennett profile, Phys. Plasmas 4, 1154 (1997).

[16] D. H. Whittum, W. M. Sharp, S. S. Yu, M. Lampe, and G. Joyce, Electron-Hose Instability in the Ion-Focused Regime, Phys. Rev. Lett. 67, 991 (1991).

[17] C. B. Schroeder, D. H. Whittum, and J. S. Wurtele, Multimode Analysis of the Hollow Plasma Channel Wakefield Accelerator, Phys. Rev. Lett. 82, 1177 (1999).

[18] A. Pukhov and J. P. Farmer, Stable Particle Acceleration in Coaxial Plasma Channels, Phys. Rev. Lett. 121, 264801 (2018).
[19] A. Pukhov, Particle-in-cell codes for plasma-based particle acceleration, CERN Yellow Reports 1, 181 (2016).

[20] A. Pukhov, Three-dimensional electromagnetic relativistic particle-in-cell code VLPL (Virtual Laser Plasma Lab), J. Plasma Phys. 61, 425 (1999).

[21] G. Penn, J.-L. Vay, R. Lehe, C. Schroeder, and E. Esarey, Beam breakup studies in a hollow plasma channel, AIP Conf. Proc. 1812, 040009 (2017).

[22] C. Huang, W. Lu, M. Zhou, C. E. Clayton, C. Joshi, W. B. Mori, P. Muggli, S. Deng, E. Oz, T. Katsouleas, M. J. Hogan, I. Blumenfeld, F. J. Decker, R. Ischebeck, R. H. Iverson, N. A. Kirby, and D. Walz, Hosing Instability in the Blow-Out Regime for Plasma-Wakefield Acceleration, Phys. Rev. Lett. 99, 255001 (2007).

[23] T. J. Mehrling, R. A. Fonseca, A. Martinez de la Ossa, and J. Vieira, Mitigation of the Hose Instability in PlasmaWakefield Accelerators, Phys. Rev. Lett. 118, 174801 (2017). 